\title{
CARBOHYDRATE COMPOSITION OF FLAX MUCILAGE AND ITS RELATION TO MORPHOLOGICAL CHARACTERS
}

\section{E.A. POROKHOVINOVA' ${ }^{1}$, A.V. PAVLOV ${ }^{1}$, N.B. BRACH ${ }^{1}$, C. MORVAN ${ }^{2}$}

\author{
1Federal Research Center the N.I. Vavilov All-Russian Institute of Plant Genetic Resources, Federal Agency of \\ Scientific Organizations, 42-44, ul. Bol'shaya Morskaya, St. Petersburg, 190000 Russia, e-mail e.porohovi- \\ nova@vir.nw.ru, n.brutch@vir.nw.ru, a.pavlov@vir.nw.ru; \\ ${ }^{2}$ Laboratoire Polymères Biopolymères Surfaces UMR, Université de Rouen/CNRS, Faculte de Sciences, UFR des \\ Sciences et Techniques, Bâtiment Pierre-Louis DULONG, Bd Maurice de Broglie, F-76821 Mont Saint Aignan, \\ Cedex, France, e-mail claudine.morvan@univ-rouen.fr
}

The authors declare no conflict of interests

Received January 22, 2016

\section{Abstract}

Mucilage of flax is a valuable product for food, medicine and biocomposites production. Each direction of use needs special characteristics of seeds, so it is necessary to describe flax polymorphism of mucilage carbohydrate composition to determine the effect of seed color and pleiotropic effects of genes controlling it on the mucilage chemical composition. The originality of the work consists in the use of lines genetic collection with identified seed color genes and methods of multivariate and nonparametric statistics to identify patterns of influence of seed color on the mucilage composition. Seed mucilage polysaccharide composition was evaluated in 29 lines and three cultivars of flax (15 lines had red-brown seeds of the wild type, 9 lines had yellow seeds and 7 lines had modified brown seeds). For some lines the genetic control of seed color was known (from 8 lines with yellow seeds 4 lines had gene $s$ 1, 4 ones had gene YSED1; 5 lines with yellow hue of seeds had gene $p f 1$ ).Water extraction of mucilage performed for 2 hours at $20{ }^{\circ} \mathrm{C}$. After freeze drying monosaccharide composition was examined by gas chromatography. Generally, mucilage contained more pectin $($ pect $=$ rhamnose $($ Rha $)+$ galacturonic acid $($ GalA), 38-64 \%) than arabinoxylans $(\mathrm{AX}=$ arabinose $(\mathrm{Ara})+$ xylose $(\mathrm{Xyl}), 10-38 \%)$. In the most of lines maximal and minimal percent of pectin was caused by rhamnogalacturonan $(\mathrm{RG} 1 \mathrm{~b}=2 \times$ Rha), except of the variety Orshanskii 2 which had an exceptionally high content of GalA and accordingly, homogalacturonan $(\mathrm{HGA}=\mathrm{GalA}-\mathrm{Rha})$. Increase of $\mathrm{AX}$ was caused by extending of the core (Xyl), but there were lines with increased branching (Ara) or proportionally increased the whole molecule. Ratio Ara:Xyl was about $0.23(0.05-0.30)$. Its extreme values did not always correspond to the AX content. Percent of RGlb was approximately twice higher than that of AX. But there were lines with more AX than RG1b. Galactose (Gal) was about $15 \%$ of mucilage sugars, fucose (Fuc) was about $3.5 \%$. In average glucose (Glc) was $3.6 \%$ of mucilage but it varied greatly (from 1.3 to $11.2 \%, \mathrm{Cv}=$ $79 \%)$. Factor analysis revealed two main factors. The factor 1 showed antagonism of AX, Ara, Xyl with pectins, Gal, and GalA. The factor 2 showed antagonism of HGA with Fuc and Ara:Xyl. Mann-Whitney $U$ rank test showed the significant decrease of AX, Ara, Xyl and conversely the increase of GalA, Gal, HGA, RG1b and RG1b:AX in brown seeds. Yellow seeds had significantly higher percent of AX, Xyl, Fuc and conversely lower percent of RG1b, HGA, GalA and Gal. Lines homozygous for the gene $s 1$ contained significantly more Glc, AX, Ara, Xyl and less Gal, RGlb, Rha, GalA at lower RGb:AX. No significant differences in the composition of mucilage for lines carrying genes $Y S E D 1$ and $p f 1$ were identified. For the first time, by nonparametric and multivariate statistics we revealed a complete difference between lines groups with brown seeds having the greatest load on the factor 1 (much pect, GalA and Gal) and yellow seeds, and also homozygotes for the gene $s 1$ having the lowest load on the factor 1 (much AX, Ara, Xyl).

Keywords: Linum usitatissimum, genetic collection, genes of seeds colours, flax mucilage, arabinoxylan, rhamnogalacturonan 1

Flax is an ancient industrial crop. Russia occupies the second place after Canada on the area of oil flax cultivation [1]. In the last decade, its unconventional use in the manufacture of bakery and pastry products, including specialized products [2-4], has sharply increased. The most important substance determining the quality of baked goods is mucilage, constituting about $5 \%$ of 
the weight of flax seeds [5]. It is also used separately as an egg white substitute [6]. Biscuits made from flax flour with a high content of arabinoxylans have a greater specific volume, openness, are more pliable and springy than similar products with a low content of arabinoxylans, and exceed the wheat flour standard according to these parameters [7]. In medicine, flaxseed mucilage is used at gastrointestinal disorders, hypercholesterolaemia, atherosclerosis, diabetes, nephritis and hormone-dependent cancer [8, 9]. It has been shown that long chains of homogalacturonan or linear rhamnogalactouronan are required for immunomodulating effect of pectins [10].

In the European Union, Canada and the USA, biocomposite materials are being intensively developed, completely consisting of plant raw materials, where fibre acts as a reinforcing component, and flax mucilage as a binder [11, 12].

Mucilage is formed in the secondary cell wall of the epidermal cells of the flax seed cover [13], and promotes their spread by the animals, adhesion to the soil, attracting soil microorganisms in the rhizosphere [14, 15], and is also used in seedling feeding [16]. In the flax mucilage, the content of polysaccharides is $83.3 \%$, with $4.6 \%$ of proteins and $11.8 \%$ of total ash [17]. The chemical composition of polysaccharides depends on the method of the mucilage extraction. It is obtained mainly from whole seeds, less often from flour [18] and seed cake [19], and then in most cases it is precipitated by a nonpolar solvent, such as ethanol or acetone, but this technique has been shown to disrupt the functional properties of mucilage [20]. No standard method has been developed to investigate the composition of carbohydrates (in contrast to proteins). The mucilage polysaccharides are analyzed using the method of size exclusion chromatography (SEC), in which the substances are separated according to their molecular weight $\left(\mathrm{M}_{\mathrm{w}}\right)$, and the homogeneity of the obtained fractions is evaluated, followed by the assessment of the monosaccharide composition of each fraction using gas chromatography [21]. More often, the monosaccharide composition of mucilage is determined without separation into fractions, as a pooled sample based on these fractions differs from the original sample in the rheological properties [21]. The composition of mucilage includes seven basic monosaccharides, xylose (Xyl), arabinose (Ara), rhamnose (Rha), galacturonic acid (GalA), galactose (Gal), fucose (Fuc) and glucose (Glc) [21].

The polysaccharides of yellow flax seeds have been most thoroughly studied [22, 23]. It has been shown that their mucilage consists of a neutral $\left(\mathrm{M}_{\mathrm{w}}\right.$ $1.16 \times 10^{6} \mathrm{~g} / \mathrm{mol}, 75.00 \%$ of the total amount $)$ and two acidic $\left(\mathrm{M}_{\mathrm{w}} 6.52 \times 10^{5}\right.$ $\mathrm{g} / \mathrm{mol}, 3.75 \%, \mathrm{M}_{\mathrm{w}} 1.35 \times 10^{4} \mathrm{~g} / \mathrm{mol}, 21.25 \%$ ) fractions, but they are also not homogeneous [19]. Neutral polysaccharides of the seed mucilage are pentosan and (galacto)arabinoxylan (AX) in a ratio of Ara:Xyl 1:5. The main polymer chain is formed by Xyl residues to which Ara and Gal are attached; also, Fuc and Rha with GalA are a part of the polysaccharide (Rha and GalA may form a part of the next fraction). Acid polysaccharides are represented by the rhamnogalacturonan 1 (RG1) pectin with a different molecular weight. Its main chain (RG1 backbone RGlb) is built from alternating hexoses of Rha and GalA; the Gal residues forming the lateral branches are linked with Rha; in addition, Fuc and Xyl are found in RG1. The fractions of flax mucilage differ in their molecular weight and the ratio of sugars.

The branching of mucilage polysaccharides is evaluated by the degree of methylation. For example, it was shown for AX that $72.5 \%$ of Xyl residues had two additional branches, $2.5 \%$ - one branch, and $25.0 \%$ are included only in the skeleton chain of AX. No one residue of Xyl was found, which would be a lateral branch. Gal, Fuc and Xyl may be terminal sugars, whereas Ara participates in the lengthening of the lateral chain [23]. Some of the Rha 
residues are found only in the skeleton of RG1, the rest have a lateral branch, whereas GalA serves exclusively as a link in the main chain of this pectin [W. Cui et al., 1994, cited from 24].

The genotype of flax significantly affects the content [25] and the composition of mucilage. Five of six samples with yellow seeds had fewer acid and more neutral polysaccharides than five of the six brown-seed varieties, but one sample from each group had the opposite mucilage composition [26]. This may indicate both a different genetic control of the yellow-seedness, and the influence of other genes on the composition of polysaccharides. Genetic control of the biosynthesis and secretion of mucilage was studied using Arabidopsis thaliana L. as a model object. Forty four genes have been sequenced that affect these processes. The detected genes were divided into four following groups: the outer integument differentiation regulators; genes of mucilage synthesis and secretion; genes involved in maintaining the structure and modification of mucilage; genes involved in the differentiation of cells secreting the mucilage [14].

To determine the patterns of the seed coloration effects on the mucilage composition, for the first time we used lines from the genetic collection with known seed color genes, as well as multi-dimensional and nonparametric statistics. For the first time it has been established that in lines carrying the $s 1$ gene, responsible for the absence of anthocyanins in the whole plant with a pleiotropic effect on the yellow coloration of the seeds, there are much more arabinoxylans and glucose in mucilage than in other lines.

The aim of this study was to establish the polymorphism of flax ( $\mathrm{Li}-$ num usitatissimum L.) for the carbohydrate composition of mucilage, as well as to evaluate the correlation between this parameter with the color of the seeds and the pleiotropic effect of the genes controlling it.

Techniques. The study used seeds of 28 lines created in the N.I. Vavilov All-Russian Institute of Plant Genetic Resources (VIR) and 3 recognized varieties grown in the fields of the VIR's laboratories in Pushkin (Leningrad region) in 2001-2007. Three varieties and 12 lines had red-brown seeds (wild type), 9 lines - yellow seeds, and 7 lines had a changed brown color of the seeds. Ten seeds from each line were soaked for 2 hours in $2 \mathrm{ml}$ of double-distilled water (20 ${ }^{\circ} \mathrm{C}$, with stirring for the first hour). The monosaccharide composition of the obtained mucilage solution (the amount of arabinose, xylose, rhamnose, galacturonic acid, fucose, galactose and glucose) was studied using a GCMS-QP5050A gas chromatograph/mass spectrometer (Shimadzu Corp., Japan) after freeze drying, methanolysis and silylation by a standard procedure [28].

The proportion of polysaccharides was calculated based on the composition of each of them: $\mathrm{AX}=\mathrm{Ara}+\mathrm{Xyl}$ (arabinoxylan), RG1b $=2 \times$ Rha (the main chain of rhamnogalacturonan 1 taking into account approximately equal molecular weight of the RG1b monomers, i.e. Rha and GalA); HGA = GalA - Rha (a linear polymer homogalactorunan was determined by the difference in total amount of GalA and the amount included in the composition of RG1b expressed by Rha), pect $=$ Rha + GalA (pectin). The ratios of Ara:Xyl and RG1b:AX were also calculated.

The data was processed using the Microsoft Excel package (primary statistics), the Statistica 7.0 programs (StatSoft, Inc., USA; factor analysis, variance analysis, Student's $t$-test), SPSS 13.0 (nonparametric statistics) [29-33].

Results. The flax varieties and lines used in the study are described in Table 1. In the samples studied, the yellow color of the seeds was controlled by different genes. The gk-103, gk-136, gk-351 and k-391 samples were homozygous for $s 1$, which inhibits the anthocyanin color of the whole plant with a pleiotropic effect (white stellate corolla, yellow anthers). The gk-351 (gk-136 × gk- 
121) line also carried the $r s 1$ gene (light yellow-brown seeds), which was hypostasized by the $s 1$ gene. In the gk-159, gk-390, gk-391 and gk-395 lines, the seed color was determined by the dominant YSED1 gene, in gk-173 - by the recessive ysed 2 gene, and not by the allelic YSED1. The yellow seedness of the gk-129 line was the result of the interaction of two genes: the main $p f-a^{d}$ gene (seeds with a yellow tinge, pink corolla, orange anthers), and yspf1 modifier (in the $p f$ $a^{d}$ yspf1 genotype the seeds are yellow). It should be noted that the gk-159 and gk-160 lines, as well as gk-390, 393 and 394 originated from common ancestors.

1. Varieties and lines of flax (Linum usitatissimum L.) from the VIR collection, used to assess the effect of seed colour on the composition of the mucilage obtained from them

\begin{tabular}{|c|c|c|c|}
\hline Cat. No & Parentage, seed color & Phenotype & Genotype \\
\hline \multicolumn{4}{|c|}{ Reb-brown seeds } \\
\hline gk-2 & 1-1 from k-48 (Altgauzen breeding station, Russia) & $\mathrm{d}$ & \\
\hline $\mathrm{gk}-22$ & 1-3-2 from k-562 (Pskov ridge, Russia) & d & \\
\hline gk-79 & 1-1-2 from k-5408 (Pechora ridge, Russia) & d & \\
\hline gk-91 & 1-1 from k-5522 (Palkinsky ridge, Russia) & $\mathrm{d}$ & \\
\hline gk-130 & 1-1 from k-6577 (Medra, Czech Republic) & $\mathrm{d}$ & \\
\hline gk-109 & 1-3-2 from k-6099 (Makovi M.A.G., Argentina) & $\mathrm{d}$ & \\
\hline $\mathrm{k}-6807$ & Orshansky 2 (Republic of Belarus) & $\mathrm{d}$ & \\
\hline gk-160 & L-2-1 from k-7659 (a biological admixture in the Bionda variety, Germany) & $\mathrm{m}$ & \\
\hline gk-125 & 1-5-1 from k-6296 (Koto, USA) & $\mathrm{m}$ & \\
\hline k-8409 & Kinelsky 2000 (Russia) & $\mathrm{m}$ & \\
\hline k-7822 & Cyan (Russia) & $\mathrm{m}$ & \\
\hline gk-132 & 1-1 from k-6608 (Currong, Australia) & $\mathrm{m}$ & \\
\hline gk-396 & 1-1-1 from i-605311 (Agt1393/02, Czech Republic) & $\mathrm{m}$ & \\
\hline gk-393 & 1-2 from i-595808 (a biological admixture in the Linola variety, Canada) & $\mathrm{m}$ & \\
\hline gk-394 & $\begin{array}{l}\text { 1-3 from i-595808 (a biological admixture in the Linola variety, Canada) } \\
\text { Ye } 11 \text { o w s e e d s }\end{array}$ & $\mathrm{m}$ & \\
\hline gk-103 & 1-4 from k-5896 (Lin 255, the Netherlands) & $\mathrm{m}$ & s1 \\
\hline gk-136 & 1-1 from k-6634 (Mermilloid, Czech Republic) & $\mathrm{m}$ & s1 \\
\hline gk-351 & $1-1$ from (gk-136 $\times$ gk-121, Russia) & $\mathrm{m}$ & $s 1, r s 1$ \\
\hline gk-159 & 1-1-1 from k-7659 (Bionda, Germany) & $\mathrm{m}$ & YSED1 \\
\hline gk-390 & 1-1 from i-595808 (a biological admixture in the Linola variety, Canada) & $\mathrm{m}$ & YSED1 \\
\hline gk-391 & 1-1-2 from i-601679 (Eyre, Australia) & $\mathrm{m}$ & YSED1 \\
\hline gk-395 & 1-1 from i-601680 (Walaga, Australia) & $\mathrm{m}$ & YSED1 \\
\hline gk-173 & 1-1 from i-548145 (Ottawa 2152, Germany) & $\mathrm{m}$ & ysed 2 \\
\hline gk-129 & $\begin{array}{l}\text { 1-2 from k-6392 (Bolley Golden, USA) } \\
\qquad \text { Ye } 11 \text { ow-brown or spot ted s e eds }\end{array}$ & $\mathrm{m}$ & $p f-a^{d}, y s p f 1$ \\
\hline gk-141 & 1-1 from k-6815 (K-6, Russia), dark yellow-brown seeds & $\mathrm{d}$ & pf 1 \\
\hline gk-143 & 1-1 from k-6917 (Versailles, France), yellow-brown seeds & $\mathrm{d}$ & $p f-a^{d}$ \\
\hline gk-176 & 1-1 from (gk-141 $\times$ gk-103), Russia, yellow-brown seeds & $\mathrm{d}$ & $p f 1, s 1$ \\
\hline gk-255 & 1-3 from (gk-121 × gk-141), Russia, yellow-brown seeds & $\mathrm{m}$ & $p f 1, r s 1$ \\
\hline gk-121 & $\begin{array}{l}\text { 1-1-1 from k-6272 (L. Dominion, Northern Ireland), light yellow-brown } \\
\text { seeds }\end{array}$ & $\mathrm{m}$ & $r s 1$ \\
\hline gk-65 & 1-3 from K-3178 (local, Tver oblast) red-brown seeds with yellow specks & $\mathrm{m}$ & oral \\
\hline gk-124 & $\begin{array}{l}\text { 1-1 from K-6284 (Stormont Mothley, Northern Ireland), red-brown seeds } \\
\text { with a yellow spot }\end{array}$ & $\mathrm{d}$ & \\
\hline & & & \\
\hline
\end{tabular}

The gk-141 and gk-143 lines with a modified brown color of the seeds were homozygous for the two different alleles of the $p f 1$ gene, i.e. $p f 1-p f 1$ and $p f$ $a^{d}$ ( $p f 1$, the seeds are dark yellow-brown; $p f-a^{d}$, seeds with a color ranging from yellow to dark yellow-brown). The gk-121 line carried the $r s 1$ gene, controlling the light yellow-brown color of the seeds. The gk-65 sample was homozygous for the oral gene, which is responsible for the appearance of yellow flecks on redbrown seeds, as well as the orange color of anthers. The gk-124 line contained the $f^{e}$ gene, which determines the presence of a yellow spot in red-brown seeds, a light blue corolla, and grey anthers. Two lines with yellow-brown seeds had a hybrid origin, such as gk-176 (gk-141 $\times$ gk-103, the $s 1$ and $p f 1$ genes) and gk255 (gk-121 $\times$ gk-141, the $p f 1$ and $r s 1$ genes) [27].

For all the samples studied, arabinoxilan averaged $26.4 \%$ of the total amount of mucilage sugars (with variations from 7.9 to $38.4 \%$ ). The highest 
content of AX was found in all lines carrying the $s 1$ gene (gk-103, ggk-136, gk351, gk-176), in two of the four lines with the YSED1 gene (gk-159, gk-391), in gk-173 carrying the ysed 2 gene and the gk-160 line with red-brown seeds. The sample from gk-351 was characterized by the maximum Ara content. In two related lines, gk-159 and gk-160, as well as in gk-132, a high content of Ara and Xyl was found. In the remaining lines, xylose was predominated in the mucilage composition. Consequently, branching was increased in the gk-351 line, a proportional increase in the size of the polysaccharide molecule occurred in gk-159, gk-160 and gk-132, and in the remaining cases the chain elongated without branching.

The minimum content of AX was seen in 5 brown-seed samples (gk-393, gk-394, k-396, k-6807 and k-8409). In the k-8409 line, this was the result of a proportional decrease in the Ara and Xyl content; whereas in the gk-394 line, the length of the main chain reduced to a greater extent, while in gk-393, gk396 and k-6807, the amount of AX decreased at the expense of lateral branches. The ratio of Ara:Xyl reflected the degree of AX branching and averaged 0.23, varying from 0.05 to 0.30 . In the yellow-seeded variant of gk-129, the degree of branching of AX was 5 times lower than in the remaining samples. Weak branching of $\mathrm{AX}$ was also observed in the arabinoxylan-deficient samples of gk393, gk-396 and k-6807. The lines with the extremal content of AX, such as gk394, gk-351 and gk-143, had the largest degree of branching (Table 2).

2. Carbohydrate composition of mucilage (\%) from seeds of different lines and varieties of flax (Linum usitatissimum L.) from the VIR collection

\begin{tabular}{|c|c|c|c|c|c|c|c|c|c|c|c|c|}
\hline Cat. No & Ara & Rha & Fuc & Xyl & GalA & Gal & Glc & HGA & pect & AX & Ara:Xyl & RGb:AX \\
\hline \multicolumn{13}{|c|}{ Reb-brown s e eds } \\
\hline $\mathrm{gk}-2$ & 6.2 & 24.4 & 3.9 & 22.6 & 25.3 & 16.2 & $1.4^{\mathrm{a}}$ & $1.0^{\mathrm{a}}$ & 49.7 & 28.8 & 0.27 & 1.70 \\
\hline gk-22 & 4.6 & 20.6 & 3.0 & 21.9 & 30.9 & 17.3 & $1.7^{\mathrm{a}}$ & 10.3 & 51.4 & 26.5 & 0.21 & $1.55^{\mathrm{a}}$ \\
\hline $\mathrm{gk}-79$ & 4.8 & 21.3 & $2.9^{a}$ & 21.9 & 30.6 & 16.9 & $1.7^{\mathrm{a}}$ & 9.4 & 51.9 & 26.6 & 0.22 & $1.60^{\mathrm{a}}$ \\
\hline gk-91 & 5.4 & 22.4 & $2.7^{\mathrm{a}}$ & 23.6 & 28.1 & 15.8 & $2.0^{\mathrm{a}}$ & 5.7 & 50.5 & 29.1 & 0.23 & $1.54^{\mathrm{a}}$ \\
\hline gk-130 & 4.0 & 20.8 & $2.9^{\mathrm{a}}$ & 16.2 & 33.4 & 15.2 & 7.5 & $12.5^{b}$ & 54.2 & 20.2 & 0.24 & 2.07 \\
\hline gk-109 & 4.7 & 19.5 & 3.1 & 19.5 & 33.1 & 18.0 & $2.0^{\mathrm{a}}$ & $13.6^{\mathrm{b}}$ & 52.7 & 24.2 & 0.24 & $1.62^{\mathrm{a}}$ \\
\hline K-6807 & 2.1 & 25.7 & $2.9^{\mathrm{a}}$ & 13.1 & $39.2^{\mathrm{b}}$ & 14.1 & 2.9 & $13.5^{b}$ & $64.9^{b}$ & 15.2 & 0.16 & 3.38 \\
\hline gk-160 & $7.7^{b}$ & 20.1 & 3.3 & $28.2^{\mathrm{b}}$ & 26.4 & 11.3 & 3.1 & 6.3 & 46.5 & $35.8^{b}$ & 0.27 & $1.12^{\mathrm{a}}$ \\
\hline gk- 125 & 4.8 & 23.6 & 3.1 & 20.0 & 28.6 & 18.7 & $1.3^{\mathrm{a}}$ & 5.0 & 52.2 & 24.8 & 0.24 & 1.90 \\
\hline k-8409 & 2.9 & 27.3 & 4.1 & 12.1 & 32.9 & 17.5 & 3.1 & 5.7 & 60.2 & 15.0 & 0.24 & 3.63 \\
\hline $\mathrm{k}-7822$ & 3.6 & 26.5 & 4.3 & 14.3 & 32.1 & 15.9 & 3.3 & 5.6 & 58.7 & 17.9 & 0.26 & 2.96 \\
\hline $\mathrm{gk}-132$ & $7.3^{\mathrm{b}}$ & 20.9 & $2.5^{\mathrm{a}}$ & $30.9^{\mathrm{b}}$ & 22.9 & 12.5 & 2.9 & $2.0^{\mathrm{a}}$ & 43.8 & $38.3^{\mathrm{b}}$ & 0.24 & $1.09^{\mathrm{a}}$ \\
\hline gk-396 & $1.5^{\mathrm{a}}$ & 26.7 & 3.4 & $8.8^{a}$ & 34.1 & $21.8^{b}$ & 3.8 & 7.4 & 60.8 & $10.2^{\mathrm{a}}$ & 0.17 & 5.23 \\
\hline gk-393 & $0.9^{\mathrm{a}}$ & $29.3^{b}$ & 3.9 & $6.9^{a}$ & 35.1 & $22.1^{b}$ & $1.7^{\mathrm{a}}$ & 5.9 & $64.4^{b}$ & $7.9^{a}$ & 0.14 & $7.44^{b}$ \\
\hline gk-394 & 2.3 & $29.4^{b}$ & 4.6 & $7.9^{a}$ & 34.6 & 19.8 & $1.4^{\mathrm{a}}$ & 5.1 & $64.0^{\mathrm{b}}$ & $10.2^{\mathrm{a}}$ & $0.29^{b}$ & 5.76 \\
\hline \multicolumn{13}{|c|}{$\mathrm{Ye} 11 \mathrm{ow}$ seeds } \\
\hline gk-103 & 6.2 & $17.1^{\mathrm{a}}$ & 3.4 & $30.9^{b}$ & $21.3^{\mathrm{a}}$ & 11.6 & 9.5 & 4.2 & $38.3^{\mathrm{a}}$ & $37.1^{\mathrm{b}}$ & 0.20 & $0.92^{\mathrm{a}}$ \\
\hline $\mathrm{gk}-136$ & 6.7 & $18.4^{\mathrm{a}}$ & 3.6 & $29.5^{b}$ & $20.2^{\mathrm{a}}$ & $10.4^{\mathrm{a}}$ & $11.2^{\mathrm{b}}$ & $1.8^{\mathrm{a}}$ & $38.6^{\mathrm{a}}$ & $36.2^{\mathrm{b}}$ & 0.23 & $1.01^{\mathrm{a}}$ \\
\hline gk-351 & $7.8^{\mathrm{b}}$ & 19.8 & $6.1^{b}$ & 26.2 & 25.7 & 11.7 & 2.7 & 5.9 & 45.5 & $34.1^{b}$ & $0.30^{\mathrm{b}}$ & $1.16^{\mathrm{a}}$ \\
\hline $\mathrm{gk}-159$ & $7.7^{b}$ & 18.9 & 3.6 & $30.7^{b}$ & $22.5^{\mathrm{a}}$ & 11.4 & 5.2 & 3.6 & 41.3 & $38.4^{\mathrm{b}}$ & 0.25 & $0.98^{\mathrm{a}}$ \\
\hline gk-390 & 4.0 & 26.7 & 3.4 & 15.8 & 32.0 & 16.8 & $1.3^{\mathrm{a}}$ & 5.4 & 58.7 & 19.8 & 0.26 & 2.69 \\
\hline gk-391 & $7.0^{\mathrm{b}}$ & 21.3 & 3.9 & $29.5^{b}$ & $21.7^{a}$ & 13.6 & 2.9 & $0.4^{\mathrm{a}}$ & 43.1 & $36.5^{b}$ & 0.24 & $1.17 \mathrm{a}$ \\
\hline gk-395 & 4.8 & 22.7 & 4.0 & 21.6 & 27.1 & 13.3 & 6.5 & 4.3 & 49.8 & 26.4 & 0.22 & 1.72 \\
\hline gk- 173 & 6.0 & 21.2 & 4.6 & $29.6^{b}$ & 25.9 & $10.4^{\mathrm{a}}$ & $2.3^{\mathrm{a}}$ & 4.7 & 47.1 & $35.6^{\mathrm{b}}$ & 0.20 & $1.19^{\mathrm{a}}$ \\
\hline gk-129 & $1.0^{\mathrm{a}}$ & 24.8 & 3.5 & 21.4 & 29.6 & 18.0 & $1.8^{\mathrm{a}}$ & 4.8 & 54.4 & 22.4 & $0.05^{\mathrm{a}}$ & 2.22 \\
\hline \multicolumn{13}{|c|}{ Yellow-brown or spotted seeds } \\
\hline $\mathrm{gk}-141$ & 5.8 & 22.7 & 3.5 & 22.2 & 26.3 & 16.0 & 3.5 & 3.6 & 48.9 & 28.1 & 0.26 & $1.62^{\mathrm{a}}$ \\
\hline gk-143 & 6.6 & 23.9 & 3.8 & 22.0 & 25.0 & 16.1 & 2.7 & $1.1^{\mathrm{a}}$ & 48.9 & 28.6 & $0.30^{\mathrm{b}}$ & 1.67 \\
\hline $\mathrm{gk}-255$ & 5.1 & 21.6 & 2.9 & 22.6 & 27.7 & 16.5 & 3.6 & 6.1 & 49.3 & 27.7 & 0.22 & $1.56^{\mathrm{a}}$ \\
\hline gk-176 & $7.0^{\mathrm{b}}$ & $17.1^{\mathrm{a}}$ & 3.4 & $28.5^{b}$ & 22.8 & $9.6^{\mathrm{a}}$ & $11.7^{\mathrm{b}}$ & 5.7 & $39.9^{a}$ & $35.5^{b}$ & 0.25 & $0.96^{\mathrm{a}}$ \\
\hline gk-121 & 3.5 & 22.5 & 3.5 & 17.5 & 33.6 & 17.1 & $2.3^{\mathrm{a}}$ & 11.0 & 56.1 & 21.0 & 0.20 & 2.15 \\
\hline gk-65 & 5.8 & 19.4 & $2.8^{\mathrm{a}}$ & 27.1 & 29.9 & 12.7 & $2.4^{\mathrm{a}}$ & 10.5 & 49.3 & 32.8 & 0.21 & $1.18^{\mathrm{a}}$ \\
\hline gk-124 & 5.7 & 25.3 & 3.1 & 21.4 & 26.3 & 16.8 & $1.4^{\mathrm{a}}$ & $0.9^{\mathrm{a}}$ & 51.6 & 27.1 & 0.27 & 1.87 \\
\hline$X_{\mathrm{av}}$ & 5.0 & 22.6 & 3.5 & 21.4 & 28.5 & 15.3 & 3.6 & 5.9 & 51.2 & 26.4 & 0.23 & 2.15 \\
\hline LSD & 0.9 & 1.6 & 0.3 & 3.3 & 2.3 & 1.6 & 1.3 & 1.7 & 3.5 & 4.2 & 0.02 & 0.72 \\
\hline$C v, \%$ & 40 & 15 & 20 & 33 & 17 & 21 & 79 & 62 & 14 & 33 & 22 & 71 \\
\hline \multicolumn{13}{|c|}{$\begin{array}{l}\text { Not e. } \mathrm{k} \text { - the VIR catalogue numbers, gk }- \text { numbers in the VIR's catalogue of the genetic collection } \\
\text { (N.I. Vavilov All-Russian Institute of Plant Genetic Resources, St. Petersburg). Ara - arabinose, Xyl - xylose, } \\
\text { Rha - rhamnose, GalA - galacturonic acid, Fuc - fucose, Gal - galactose, Glc - glucose, HGA - homoga- } \\
\text { lacturonan (HGA = GalA - Rha), pect - pectin (pect = Rha }+ \text { GalA), AX - arabinoxylan (AX = Ara }+ \text { Xyl); } \\
\text { Ara:Xyl - the ratio of arabinose and xylose, RGlb:AX - the ratio of rhamnogalacturonan } 1 \text { and arabinoxylan. } \\
X_{\text {av. }}-\text { the mean for the whole sample, } C v-\text { coefficient of variation, a - minimum values, b - maximum values. }\end{array}$} \\
\hline
\end{tabular}


On average in the sample, pectins accounted for $51.2 \%$ of the total amount of mucilage sugars (from 38.3 to $64.9 \%, C v=14 \%$ ) (see Table 2). Brown-seeded samples of the gk-393, gk-394, gk-396, k-6807, k-8409, k-7820 lines and yellow-seeded sample of gk-390 produced the greatest amount of pectins. Of interest, the gk-393, gk-394 and gk-390 lines originated from the same variety, Linola. Yellow-seeded lines with the $s 1$ gene (gk-136, gk-103, gk-176) and the YSED1 gene (gk-159) were characterized by the lowest content of pectins. Both the maximum and the minimum amounts of pectins were caused by the content of RGlb (with the exception of the sample of k-6807, in which a high proportion of GalA and, accordingly, HGA was observed). An increased amount of HGA was also found in the gk-109 and gk-130 lines with red-brown seeds and in the gk-121 line with light yellow-brown seeds. The minimum proportion of HGA in mucilage was found in the wild-type gk-2 line, the yellowseeded sample of the gk-391 line, and in the lines with a modified color of seeds, such as gk-124 and gk-143.

In most lines, the content of RGlb in mucilage was higher than that of AX: the RGIb:AX ratio averaged 2.2, with a median of $\mathrm{Md}=1.6$. However, this parameter varied greatly $(C v=71 \%)$, because there were samples (gk-393, gk-394, gk396), in which the amount of RG1b was 7.4-5.5 times higher than that of AX, while in the yellow-seeded lines carrying the $s 1$ gene (gk-103, k-176, k-136) and the YSED1 gene (gk-159), RG1b and AX were present in equal portions (see Table 2). Such a high variability in the ratio of acid and basic polysaccharides in the mucilage from flax seeds is consistent with the data presented by W. Cui et al. [26].

The portion of galactose in mucilage on average in the sample was $15.3 \%$ of the total amount of mucilage carbohydrates with a variation from 9.6 to $22.1 \%$. The maximum content of Gal was found in the gk-393, gk-394, gk125 lines with brown seeds, while the minimum in the yellow-seeded samples of the gk-173 (the ysed2 gene), gk-136, gk-103, gk-176, gk-351 (the $s 1$ gene), gk159 (the YSED1 gene) lines and in the gk-160 line with brown seeds. Fucose accounted for an average of $3.5 \%$ (ranging from 2.5 to $6.1 \%$ ). The highest values of this parameter were observed for yellow seeds from the gk-351 (the $s 1, r s 1$ genes) and gk-173 (the ysed 2 gene) lines, and brown seeds from the gk-394, k7822 lines, while the lowest values were seen for the gk-132, gk-91, gk-130, gk79, gk-22, k-255, gk-65 and k-6807 lines. The glucose content varied greatly within the sample studied $(C v=79 \%)$, averaging to $3.6 \%(\mathrm{Md}=2.7 \%)$, whereas for the gk-130 line and the yellow-seeded gk-159 and gk-395 lines (the YSED1 gene), this parameter was almost twice as high, i.e. $>5 \%$, and in the lines carrying the $s 1$ gene (gk-103, gk-136 and gk-176), it reached 9.5-11.7\% (see Table 2). In the observed disproportion, the origin of Glc can be associated with the xyloglucans of the primary cell wall, which is more fragile in the mutant lines, and also with starch extracted from the deeper layers of the seed (endosperm).

Using the principal component analysis, we have revealed two factors influencing the composition of mucilage in the lines studied (Fig.). The first factor (F1) determined the ratio of the two main polysaccharides in the mucilage, i.e. of pectins and pentosans (AX). The analysis identified the antagonism between AX, Ara, Xyl, on the one hand, and between pect, GalA, Gal - on the other. This factor determined about $60 \%$ of the total variability. Based on this, two groups of lines were identified: with the highest percentage of AX, such as yellow-seeded samples of the gk-136, gk-103, gk-159, gk-391, k-351, gk-173 lines, their relatives (gk-160 and gk-176), as well as the gk-132 line; with the highest proportion of pectins, such as wild-type samples of the gk-393, gk-394, gk-396, k-6807, k-8409, k-7822 lines and yellow-seeded samples of the gk-129, gk-390 lines. The second factor (F2) determined the ratio of HGA with Fuc and 
Ara:Xyl. There was an antagonism revealed between HGA, on the one hand, and Fuc, Ara:Xyl, on the other hand. F2 was responsible for about $15 \%$ of the total variability, with the samples divided into the following groups: the first one with the highest content of HGA (k-6807, gk-130, rk-65, gk-22, gk-79, gk-121, gk109) and the smallest ratio of Ara:Xyl (gk-129, gk-103); the second group with the largest percentage of Fuc (gk-351), the maximum ratio of Ara:Xyl (gk-2, gk-143, gk-394) and with the lowest content of HGA (gk-124, gk-391).

A

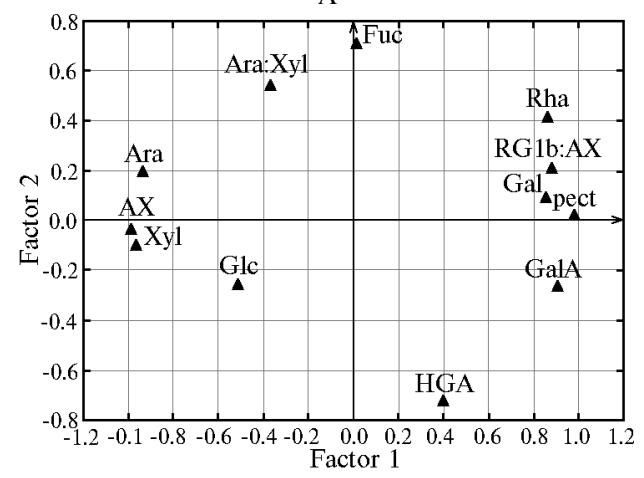

B

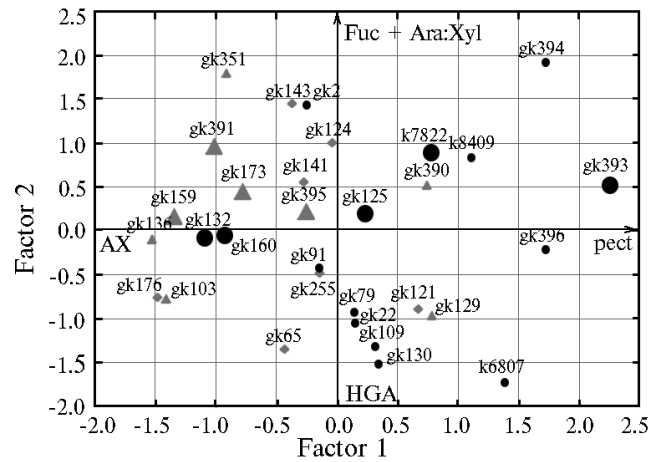

The distribution of sugars and polysaccharides in the mucilage of seeds in the studied lines of flax ( $\mathrm{Li}$ num usitatissimum L.) (A) and these very lines according to these parameters (B) using the principal component analysis: Factor 1 - a ratio of pectins and pentosans, Factor $2-$ a ratio of HGA with Fuc and Ara:Xyl; Ara - arabinose, Xyl - xylose, Rha - rhamnose, GalA - galacturonic acid, Fuc - fucose, Gal - galactose, Glc - glucose, HGA - homogalacturonan (HGA = GalA - Rha), pect - pectin $($ pect $=$ Rha + GalA $), A X-$ arabinoxylan $(A X=A r a+X y l), R G 1 b-$ ramnogalacturonan 1. Circles, triangles and diamonds (B) mark the lines with red-brown, yellow, yellow-brown or spotted seeds, respectively. Large icons correspond to the lines with large seeds.

Within the system of two factors, two groups of lines were distinguished, where the first group consisted of the yellow-seeded lines (gk-136, gk-103, gk159, gk-391, gk-351, gk-173) and their relatives (gk-160, rgk-176) with the highest percentage of AX; the second group included the brown-seeded lines (gk-22, gk-79, gk-109, gk-130) and a sample of the gk-121 line with the highest proportion of pectins and an increased content of HGA in them, as well as the gk-129 line with a low Ara:Xyl ratio.

The multi-dimensional scaling method made it possible to divide the characteristics and lines between groups equal to those obtained by the factor analysis (data not shown).

To determine the effect of morphological features of flax on the carbohydrate composition of mucilage, we used the rank Mann-Whitney U test (Table 3). A significant decrease in the proportion of arabinoxylan (percentages of AX, Ara, Xyl) and increase in pectins (GalA, Gal, HGA, pect, RGlb:AX), as well as an increased factor loadings of $F 1$ were found in mucilage extracted from brown seeds compared to that from the rest ones. In yellow seeds, the content of AX, Xyl, and Fuc was significantly higher, while that of pect, HGA, GalA and Gal lower, also, low loadings of F1 were revealed. In oil flax, a small yet significant excess of the Fuc portion was noted (Table 3).

Lines homozygous for the $s 1$ gene had significantly higher values for Glc and arabinoxylan (AX, Ara, Xyl) and lower values for Gal and pectins (pect, Rha, GalA, RGb:AX), as well as low values of factor loadings by F1. We revealed no significant differences in the mucilage composition for the lines carrying the YSED1 and $p f 1$ genes (see Table 3).

The analysis of variance and Student's $t$-test confirmed the conclusions drawn based on the rank Mann-Whitney U test (data not shown). 
3. A comparison of the carbohydrate composition of mucilage (\%) in the lines of flax (Linum usitatissimum L.) with various seed coloring and type of use (using the Mann-Whitney U test)

\begin{tabular}{|c|c|c|c|c|c|c|c|c|c|c|c|c|c|c|}
\hline Characteristic & Presence & $n$ & Ara & Xyl & Rha & GalA & Fuc & Gal & Glc & HGA & pect & $\mathrm{AX}$ & RGb:AX & F1 \\
\hline \multirow[t]{3}{*}{ Oil use } & Yes & 20 & $4.8 \pm 0.5$ & $21.6 \pm 1.9$ & $22.9 \pm 0.8$ & $28.2 \pm 1.1$ & $3.7 \pm 0.2$ & $15.2 \pm 0.8$ & $3.6 \pm 0.6$ & $5.3 \pm 0.6$ & $51.1 \pm 1.8$ & $26.4 \pm 2.3$ & $2.35 \pm 0.41$ & $1.15 \pm 0.53$ \\
\hline & No & 11 & $5.2 \pm 0.4$ & $21.2 \pm 1.2$ & $22.2 \pm 0.8$ & $29.2 \pm 1.4$ & $3.2 \pm 0.1$ & $15.6 \pm 0.7$ & $3.5 \pm 1.0$ & $7.0 \pm 1.5$ & $51.3 \pm 1.8$ & $26.3 \pm 1.6$ & $1.78 \pm 0.18$ & $0.68 \pm 0.48$ \\
\hline & $\mathrm{p}$ & & 0.97 & 0.71 & 0.68 & 0.80 & $0.04^{*}$ & 0.71 & 0.46 & 0.48 & 0.80 & 0.84 & 0.93 & 0.90 \\
\hline \multicolumn{15}{|l|}{$\begin{array}{l}\text { Seeds: } \\
\text { brown }\end{array}$} \\
\hline brown & Yes & 15 & $4.2 \pm 0.5$ & $17.9 \pm 1.9$ & $23.9 \pm 0.9$ & $31.2 \pm 1.1$ & $3.4 \pm 0.2$ & $16.9 \pm 1.1$ & $2.7 \pm 0.4$ & $7.3 \pm 1.0$ & $55.1 \pm 1.7$ & $22.0 \pm 2.4$ & $2.84 \pm 0.50$ & $0.52 \pm 0.25$ \\
\hline \multirow[t]{2}{*}{ No } & Нет & 16 & $5.7 \pm 0.4$ & $24.8 \pm 1.2$ & $21.5 \pm 0.7$ & $26.1 \pm 1.0$ & $3.7 \pm 0.2$ & $13.9 \pm 1.0$ & $4.4 \pm 0.9$ & $4.6 \pm 0.8$ & $47.5 \pm 1.5$ & $30.5 \pm 1.5$ & $1.50 \pm 0.13$ & $-0.48 \pm 0.19$ \\
\hline & $\mathrm{p}$ & & $0.02^{*}$ & $0.01^{*}$ & 0.08 & $0.002^{*}$ & 0.18 & $0.02^{*}$ & 0.15 & $0.03^{*}$ & $0.00^{*}$ & $0.01^{*}$ & $0.03^{*}$ & $0.01^{*}$ \\
\hline \multirow[t]{3}{*}{ yellow } & Yes & 9 & $5.7 \pm 0.7$ & $26.1 \pm 1.8$ & $21.2 \pm 1.0$ & $25.1 \pm 1.3$ & $4.0 \pm 0.3$ & $13.0 \pm 0.9$ & $4.8 \pm 1.2$ & $3.9 \pm 0.6$ & $46.3 \pm 2.3$ & $31.8 \pm 2.3$ & $1.45 \pm 0.21$ & $-0.63 \pm 0.29$ \\
\hline & Нет & 22 & $4.6 \pm 0.4$ & $19.5 \pm 1.4$ & $23.2 \pm 0.7$ & $29.9 \pm 0.9$ & $3.4 \pm 0.1$ & $16.3 \pm 0.6$ & $3.1 \pm 0.5$ & $6.7 \pm 0.8$ & $53.2 \pm 1.4$ & $24.2 \pm 1.8$ & $2.44 \pm 0.36$ & $0.26 \pm 0.20$ \\
\hline & $\mathrm{p}$ & & 0.10 & $0.03^{*}$ & 0.14 & $0.01^{*}$ & $0.03^{*}$ & $0.02 *$ & 0.28 & $0.02^{*}$ & $0.02^{*}$ & $0.03^{*}$ & 0.06 & $0.03^{*}$ \\
\hline \multicolumn{15}{|c|}{ Homozygous for the gene: } \\
\hline s1 & Yes & 4 & $6.9 \pm 0.3$ & $28.8 \pm 1.0$ & $18.1 \pm 0.7$ & $22.5 \pm 1.2$ & $4.1 \pm 0.7$ & $10.8 \pm 0.5$ & $8.8 \pm 2.1$ & $4.4 \pm 0.9$ & $40.6 \pm 0.6$ & $35.7 \pm 0.6$ & $1.01 \pm 0.05$ & $-1.33 \pm 0.14$ \\
\hline \multirow[t]{2}{*}{ No } & Нет & 27 & $4.7 \pm 0.4$ & $20.3 \pm 1.3$ & $23.3 \pm 0.6$ & $29.4 \pm 0.8$ & $3.5 \pm 0.1$ & $16.0 \pm 0.6$ & $2.8 \pm 0.3$ & $6.1 \pm 0.7$ & $52.8 \pm 1.2$ & $25.0 \pm 1.6$ & $2.32 \pm 0.30$ & $0.20 \pm 0.18$ \\
\hline & $\mathrm{p}$ & & $0.01^{*}$ & $0.02^{*}$ & $0.003^{*}$ & $0.01^{*}$ & 0.32 & $0.01 *$ & $0.01^{*}$ & 0.56 & $0.003^{*}$ & $0.02^{*}$ & $0.003^{*}$ & $0.003^{*}$ \\
\hline \multirow[t]{3}{*}{ YSED 1} & Yes & 4 & $5.9 \pm 0.9$ & $24.4 \pm 3.5$ & $22.4 \pm 1.6$ & $25.8 \pm 2.4$ & $3.7 \pm 0.1$ & $13.8 \pm 1.1$ & $4.0 \pm 1.2$ & $3.4 \pm 1.1$ & $48.2 \pm 3.9$ & $30.3 \pm 4.4$ & $1.64 \pm 0.38$ & $-0.46 \pm 0.46$ \\
\hline & Нет & 27 & $4.8 \pm 0.4$ & $21.0 \pm 1.3$ & $22.7 \pm 0.7$ & $28.9 \pm 0.9$ & $3.5 \pm 0.1$ & $15.6 \pm 0.6$ & $3.5 \pm 0.6$ & $6.3 \pm 0.7$ & $51.6 \pm 1.4$ & $25.8 \pm 1.7$ & $2.23 \pm 0.31$ & $0.07 \pm 0.19$ \\
\hline & $\mathrm{p}$ & & 0.35 & 0.44 & 1.00 & 0.22 & 0.26 & 0.29 & 0.60 & 0.08 & 0.41 & 0.38 & 0.60 & 0.32 \\
\hline \multirow[t]{3}{*}{$p f 1$} & Yes & 5 & $5.1 \pm 1.1$ & $23.3 \pm 1.3$ & $22.0 \pm 1.3$ & $26.3 \pm 1.2$ & $3.4 \pm 0.1$ & $15.2 \pm 1.5$ & $4.6 \pm 1.8$ & $4.3 \pm 0.9$ & $48.3 \pm 2.3$ & $28.4 \pm 2.1$ & $1.61 \pm 0.2$ & $-0.30 \pm 0.36$ \\
\hline & Нет & 26 & $4.9 \pm 0.4$ & $21.1 \pm 1.5$ & $22.8 \pm 0.7$ & $29.0 \pm 1.0$ & $3.6 \pm 0.1$ & $15.3 \pm 0.7$ & $3.4 \pm 0.5$ & $6.2 \pm 0.7$ & $51.7 \pm 1.5$ & $26.0 \pm 1.8$ & $2.26 \pm 0.32$ & $0.06 \pm 0.20$ \\
\hline & $\mathrm{p}$ & & 0.63 & 0.52 & 0.96 & 0.22 & 1.00 & 0.96 & 0.24 & 0.39 & 0.26 & 0.67 & 0.67 & 0.49 \\
\hline $\begin{array}{l}\text { lacturonan }(\mathrm{HGA}= \\
\text { nogalacturonan } 1 \text { and } \\
\text { availability or lack of } \\
* \text { Differences between }\end{array}$ & native group & & zed pa & ater are & ficant. & 0.05 & & 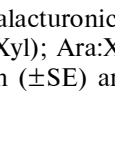 & & - fucos & hood & iilarity & ween alte & $\begin{array}{l}\text { SA - homo } \\
\text { ratio of rhe } \\
\text { ive groups }\end{array}$ \\
\hline
\end{tabular}


Therefore, the studied lines of flax have the wide polymorphism in terms of the composition of mucilage. Yellow seeds (the $s 1$ gene) compared to the brown ones contain on average a higher level of neutral polysaccharides (arabinoxylans), while brown ones were higher in acid polysaccharides (pectins). However, there are lines that have the opposite ratio of acid and basic fractions of the mucilage. The impact of other genes (YSED1 and $p f 1$ ), controlling the changed color of seeds in flax, on the composition of extracted mucilage was not revealed. For the first time, we demonstrated the way to use the results of determination of the factor loadings on the lines as an independent complex characteristic, allowing sampling by a set of characteristics.

\section{REFERE N C ES}

1. FAOSTAT domains: «crops processed», element: «Area harvested», crops: linseed, flax. Rezhim dostupa: http://faostat3.fao.org/download/Q/QC/E . Bez daty.

2. Kireeva M.S., Merkulova M.I., Porokhovinova E.A., Krasil'nikov V.N. II Manyakinskie chteniya [Proc. II Manyakin readings]. Omsk, 2013: 316-322 (in Russ.).

3. Li p i lina E., G a n j i V. Incorporation of ground flaxseed into bakery products and its effect on sensory and nutritional characteristics - a pilot study. Journal of Foodservice, 2009, 20: 5259 (doi: 10.1111/j.1748-0159.2008.00124.x).

4. S inger F.A.W., Taha F.S., Moha med S.S., Gibriel A., E 1 - N aw aw y M. Preparation of mucilage/protein products from flaxseed. Am. J. Food Technol., 2011, 6: 260-278 (doi: 10.3923/ajft.2011.260.278).

5. B h a t y S. Further compositional analysis of flax: mucilage, trypsin inhibitors and hydrocyanic acid. J. Am. Oil Chem. Soc., 1993, 70: 899-904 (doi: 10.1007/BF02545351).

6. Kaewmanee T., Bagnasco L., Benjakul S., Lanteri S., Morelli C.F., $\mathrm{S}$ pe ranza G., C o s u 1 i c h M.E. Characterization of mucilages extracted from seven Italian cultivars of flax. Food Chem., 2014, 148: 60-69 (doi: 10.1016/j.foodchem.2013.10.022).

7. K i re e v M.S. Funktsional'no-tekhnologicheskie svoistva semyan l'na i razrabotka tekhnologii muchnykh konditerskikh izdelii spetsializirovannogo naznacheniya na ikh osnove. Avtoreferat kandidatskoi dissertatsii [Flax seed functional and technological properties, and flour-based technology for special confectionery. PhD Thesis]. St. Petersburg, 2014 (in Russ.).

8. Murav'e va D.A., S a m y lin a I.A., Ya k ov lev G.P. Farmakognoziya [Pharmacognosy]. Moscow, 2002: 103-105 (in Russ.).

9. Rubial M., Gutierrez C., Verdugo M., Shene C., Sineiro J. Flaxseed as a source of functional ingredients. J. Soil Sci. Plant Nutr., 2010, 10(3): 373-377.

10. Ov o d o v Yu.S. Bioorganicheskaya khimiya, 1998, 24(7): 483-501 (in Russ.).

11. Alix S., Marais S., Morvan C., Lebrum L. Biocomposite materials from flax plants: Preparation and properties. Composites Part A: Applied Science and Manufacturing, 2008, 39: 17931801 (doi: 10.1016/j.compositesa.2008.08.008).

12. Pay ne 1 F., M orvan C., M a rais S., Le bru n L. Improvement of the hydrolytic stability of new flax-based biocomposite materials. Polym. Degrad. Stabil., 2013, 98(1): 190-197 (doi: 10.1016/j.polymdegradstab.2012.10.010).

13. Attoumbré J., Abarchi B., Laoualy M., Bienaimé C., Dubois F., Baltora$\mathrm{R}$ o s s e $\mathrm{t}$ S. Investigation of lignan accumulation in developing Linum usitatissimum seeds by immunolocalization and HPLC. Phytochem. Lett., 2011, 4: 194-198 (doi: 10.1016/j.phytol.2011.03.004).

14. We ste rn T. The sticky tale of seed coat mucilages: production, genetics, and role in seed germination and dispersal. Seed Sci. Res., 2012, 22: 1-25 (doi: 10.1017/S0960258511000249).

15. Y a ng X.J., B a s k i n c J.M., B a s k i n c C.C., H u a ng Z. More than just a coating: ecological importance, taxonomic occurrence and phylogenetic relationships of seed coat mucilage. Perspectives in Plant Ecology, Evolution and Systematics, 2012, 14: 434-442 (doi: 10.1016/j.ppees.2012.09.002).

16. Yang X.J., B askinc C.C., B a skinc J.M., Z hang W., Huang Z. Degradation of seed mucilage by soil microflora promotes early seedling growth of a desert sand dune plant. Plant Cell Environ., 2012, 35: 872-883 (doi: 10.1111/j.1365-3040.2011.02459.x).

17. M a z z a G., B i li a d e ris C. Functional properties of flax seed mucilage. J. Food Sci., 1989, 54: 1302-1305 (doi: 10.1111/j.1365-2621.1989.tb05978.x).

18. Goh K.K.T., Pinder D.N., Hall C.E., He mar Y. Rheological and light scattering properties of flaxseed polysaccharide aqueous solutions. Biomacromolecules, 2006, 7: 3098-3103 (doi: 10.1021/bm060577u).

19. Warrand J., Michaud P., Picton L., Muller G., Courtois B., Ralainirin a R., C o u rto u s. Flax (Linum usitatissimum) seed cake: a potential source of high molec- 
ular weight arabinoxylans? J. Agric. Food Chem., 2005, 53: 1449-1452 (doi: 10.1021/jf048910d).

20. Wang Y., Li D., Wang L.-J., Li S.-J., Adhika ri B. Effect of drying methods on the functional properties of flaxseed gum powders. Carbohyd. Polym., 2010, 81: 128-133 (doi: 10.1016/j.carbpol.2010.02.005).

21. N a r a n R., Che n G., C a r p it a N. Novel rhamnogalacturonan I and arabinoxylan polysaccharides of flax seed mucilage. Plant Physiol., 2008, 148: 132-141 (doi: 10.1104/pp.108.123513).

22. Warrand J., Michaud P., Picton L., Muller G., Courtois B., Ralaini r in a R., C o u rt o u s J. Large-scale purification of water soluble polysaccharides from flaxseed mucilage, and isolation of a new anionic polymer. Chromatographia, 2003, 58: 331-335 (doi: 10.1365/s10337-003-0060-4).

23. Warrand J., Michaud P., Picton L., Mul1er G., Courtois B., Rala inirina R., Courtous J. Structural investigations of the neutral polysaccharide of Linum usitatissimum L. seed mucilage. Int. J. Biol. Macromol., 2005, 35: 121-125 (doi: 10.1016/j.ijbiomac.2004.12.006).

24. M a z z a G., O o m a h B.D. Flaxseed, dietary fiber, and cyanogens. In: Flaxseed in human nutrition. S.C. Cunnne, L.U. Thompson (eds.). Champaign, Illinois, 1995: 57-82.

25. D i e d e ri ch se n A., R a n e y J.P., D u g u i d S.D. Variation of mucilage in flax seed and its relationship with other seed characters. Crop Sci., 2006, 46: 365-371 (doi: 10.2135/cropsci2005.0146).

26. Cu i W., K e n a s c huk E., M a z z a G. Influence of genotype on chemical composition and rheological properties of flaxseed gums. Food Hydrocolloids, 1996, 10: 221-227 (doi: 10.1016/S0268-005X(96)80038-5).

27. P o r o k hovi nova E.A. Trudy po prikladnoi botanike, genetike i selektsii, 2011, 167: 159-183 (in Russ.).

28. Goubet F., Bourland T., Girault R., Alexandre C., Vandevelde M.C., Morvan C. Structural features of galactans from flax fibres. Carbohydr. Polym., 1995, 27: 221-227 (doi: 10.1016/0144-8617(95)00063-D).

29. Terent'ev P.V., Rostova N.S. Praktikum po biometrii [Practical works in biometry]. Leningrad, 1977 (in Russ.).

30. Ivanter E.V., Korosov A.V. Vvedenie $v$ kolichestvennuyu biologiyu [Introduction to quantitative biology]. Petrozavodsk, 2003 (in Russ.).

31. N as ledov A.D. Matematicheskie metody psikhologicheskogo issledovaniya. Analiz i interpretatsiya dannykh [Mathematics for psychological research. Data analysis and interpretation]. St. Petersburg, 2012 (in Russ.).

32. Electronic Statistics Textbook. StatSoft, Inc., Tulsa, OK, 2013. Aavailable http://www.statsoft.com/textbook/. No date.

33. S o k a l R.R., R o h lf F.J. Biometry: the principles and practice of statistics in biological research. Freeman, NY, 1995. 